\title{
An Overview on Study of Identification of Driver Behavior Characteristics for Automotive Control
}

\author{
Na Lin, ${ }^{1}$ Changfu Zong, ${ }^{1}$ Masayoshi Tomizuka, ${ }^{2}$ Pan Song, ${ }^{1,2}$ Zexing Zhang, ${ }^{1}$ and Gang Li ${ }^{3}$ \\ ${ }^{1}$ State Key Laboratory of Automotive Simulation and Control, Jilin University, Changchun 130022, China \\ ${ }^{2}$ Department of Mechanical Engineering, University of California, Berkeley, CA 94720, USA \\ ${ }^{3}$ Liaoning University of Technology, Jinzhou 121001, China \\ Correspondence should be addressed to Changfu Zong; zong.changfu@ascl.jlu.edu.cn
}

Received 29 October 2013; Revised 24 January 2014; Accepted 7 February 2014; Published 17 March 2014

Academic Editor: Hamid R. Karimi

Copyright (c) $2014 \mathrm{Na}$ Lin et al. This is an open access article distributed under the Creative Commons Attribution License, which permits unrestricted use, distribution, and reproduction in any medium, provided the original work is properly cited.

Driver characteristics have been the research focus for automotive control. Study on identification of driver characteristics is provided in this paper in terms of its relevant research directions and key technologies involved. This paper discusses the driver characteristics based on driver's operation behavior, or the driver behavior characteristics. Following the presentation of the fundamental of the driver behavior characteristics, the key technologies of the driver behavior characteristics are reviewed in detail, including classification and identification methods of the driver behavior characteristics, experimental design and data acquisition, and model adaptation. Moreover, this paper discusses applications of the identification of the driver behavior characteristics which has been applied to the intelligent driver advisory system, the driver safety warning system, and the vehicle dynamics control system. At last, some ideas about the future work are concluded.

\section{Introduction}

In the driver-vehicle-road closed-loop system, the driver plays the role of not only the controller, but also the major evaluator of the quality of the vehicle path-following. Due to variant of driving experiences, emotions, driving preferences, and so on between drivers, the driver becomes the weakest part in the driver-vehicle-road closed-loop system. And different drivers display distinct behaviors; that is to say, every driver has his/her unique driving characteristic (also referred to as driving style). To improve the performance of the driver-vehicle-road closed-loop system, research on the driver characteristics includes (1) driver characteristics identification based on head movement and facial features, such as the eye movement recognition, which identifies the driver's driving status (fatigue/drunk/drowsy/distracted driving) and warns the driver in order to improve the active safety performance [1-3]; (2) physiological-based and psychologicalbased driver characteristics identification, which enhances the human-machine interactive performance and/or improve the driver's operation comfort [4-7]; (3) driver characteristics identification based on driver's operation behavior, which detects abnormal driving behavior and then alarm the driver, design control method for driving comfort, and/or design the human-centered driving assistance systems [8-11]; and (4) the research on the driver characteristics based on dynamics simulations of the driver-vehicle-road closed-loop system, which is aimed at the optimal design on dynamic performance of the closed-loop system [12-16].

This paper focuses on the identification of driver characteristics based on driver's operation behavior, namely, the identification of driver behavior characteristics. In the current research, the identification is generally realized based on the measured real-time driver behavior and vehicle states, or by monitoring driver's head movements and/or facial expressions. It is believed that on the completion of the identification of driver behavior characteristics, the following task can be performed: (1) the vehicle would follow the current driver's operation, trigger the appropriate driving assistant device, in order to achieve smooth transition of the semiautonomous human-machine control modes, and to realize coordination of driver's operation and automatic control [17]; (2) the parameters of electronic control system would be automatically modified [18], or appropriate characteristics of the ideal reference models established are chosen to achieve 
the ideal dynamic response of the vehicle, driver adaptive control, and personalized driving $[19,20]$; (3) the systems that real-time monitor and assess the driver's driving behaviors and driving status (fatigue/drunk/drowsy/distracted driving) can early detect possible operating errors and warn the driver to avoid traffic accidents in visual, auditory, or tactile approaches $[8,21]$. Since identification of the driver behavior characteristics is of great importance to improve automotive active safety and to achieve intelligent driving, more and more researchers have been committed to studying the related fields, mainly Ford [12, 13, 18, 22, 23], Nissan Institute [17, 24, 25], Columbia University [8], Vienna University of Technology [26], Nagoya University [27-32], Tsinghua University [10, 33-36], Jilin University [20, 37-40], and Chinese University of Hong Kong [41].

This paper is organized as follows: Section 2 introduces the fundamental of the driver behavior characteristics. Section 3 reviews key technologies about driver characteristics identification. In Section 4, applications of the identification of the driver behavior characteristics are introduced. Conclusions are drawn in Section 5 and future research work is suggested.

\section{The Driver Behavior Characteristics}

In driving a vehicle, the driver makes his/her driving intentions and selects a series of operation behaviors that are most suitable for the current driving conditions. Even very simple driving intentions (long-term driving intention) can be subdivided into a series of simpler driving operation behaviors (short-term driving behavior); that is, the driving intention is achieved by a series of driving behaviors [37]. It is widely accepted that the driving behaviors vary between drivers according to their ages, genders, ethnicities, driving experiences, emotions, and so forth [17, 42-44]. Even for the same driver, driving behavior may alter from situation to situation [27], which can be attributed to the driver behavior characteristics. Wahab et al. [45] believe that the differences of each driver in the driver characteristics are due to the way drivers' subconscious mind works and responds, and the conversion from subconscious to conscious minds would also generate unique responds on how the brains work.

There are a lot of literature investigating the uniqueness of driving behaviors in vehicles and the possibility to use it for identifying the driver characteristics, with the objectives to achieve safer and personalized driving, to detect driver's abnormal operation and then alarm, to realize integration between the driver and the electronic control systems, or to build identification models of the driver behavior characteristics [22, 28, 46-53]. For example, data collected by a set of vehicle sensors can be processed by certain recognition methods to recognize a series of driving maneuvers, and the parameters of these driving maneuvers could be extracted and used to classify driver characteristics or evaluate driver's abilities [47]. Figure 1 gives the structure of neural net pattern recognition for classifying driving behavior during carfollowing condition. Closing-in is referred to the following vehicle close to the leading vehicle, and falling-behind is

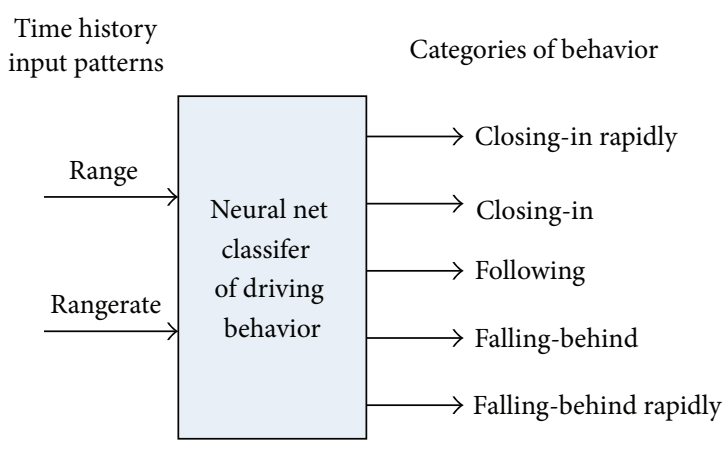

Figure 1: Model by MacAdam et al., adopted from [48].

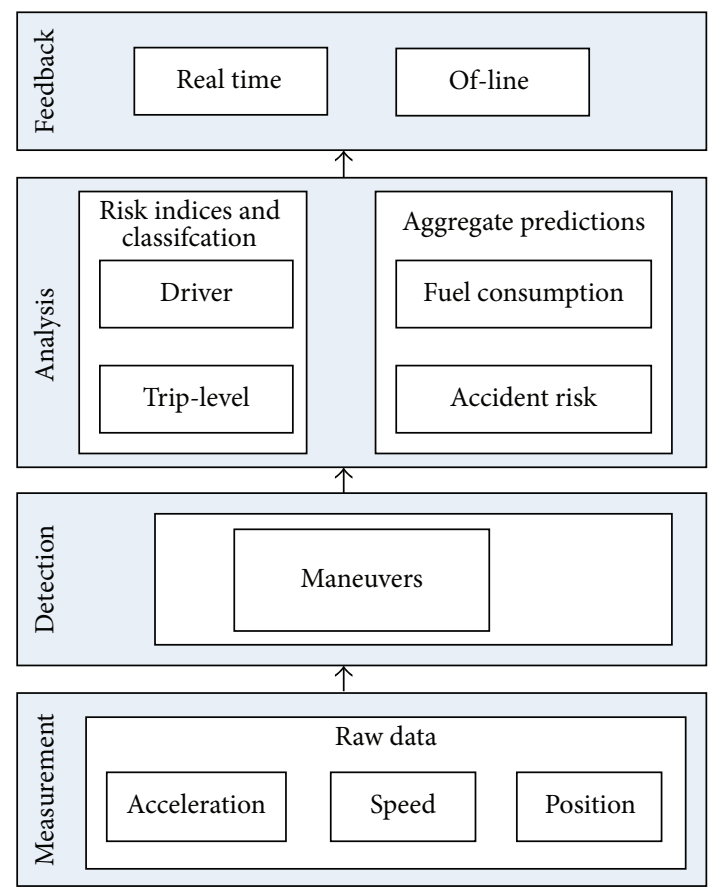

FIGURE 2: Overall framework of the IVDR system, adopted from $[50]$.

referred to the leading vehicle away from the following vehicle.

Some monitoring and analysis systems are also developed and available to research driving behaviors and finally driver behavior characteristics are obtained. Tomer Toledo develops the in-vehicle data recorder (IVDR) system [49, 50] to monitor and analyze driving behaviors, as shown in Figure 2. It can be seen from the figure that this system can identify various maneuvers that occur in the measurements, and the results can be used to evaluate risk indices that show the safety on the overall trip and to classify the drivers' characteristics. Some other relevant reviews can be found in [51, 52].

\section{Key Technologies for Identification of Driver Behavior Characteristics}

According to previous studies, identification of the driver behavior characteristics is modeled based on certain pattern 
recognition methods by use of simulation or field test data. Therefore, the selection of pattern recognition methods, experimental design, and data acquisition are of great importance to build identification models of the driver behavior characteristics. In general, the driver behavior characteristics need to be classified before identified.

\subsection{Classification of Driver Behavior Characteristics. Fuzzy} control theory and K-means algorithm are generally used to cluster the feature parameters that reflect the driver behavior characteristics, in order to achieve classification of the driver behavior characteristics. To reach a feasible classification of the driver behavior characteristics, it is necessary to take the following aspects into consideration [33]. First, it is essential to select feasible metrics which can describe the driver characteristics. It should be noted that the metrics that represent the driver characteristics are chosen on purpose so that they can be expressed by use of the defined and measurable parameters. Second, the classification results are directly affected by the clustering method selected. For example, being a kind of learning method without surveillance, the K-means clustering algorithm has rapid convergence speed and concise structure; however, measurement errors and uncertainties are ignored. Third, the sample size of the parameters affects the clustering results. In general, an increase in the amount of data (or number of drivers) would improve the accuracy of the classification.

$\mathrm{Lu}$ et al. propose that the driving behaviors can be divided into four categories with respect to the handling limit conditions (conditions beyond the limits of tire adhesion): cautious, average, expert, and reckless [12]. A cautious driver is interpreted as someone who usually drives without frequent aggressive maneuvers, for example, rapid steering, high speed, and quickly stepping on the pedal. An average driver features driving a car with a higher level of handling risk factor (HRF, the parameter that evaluates how a driving condition is close to the handling limit) than a cautious driver does. An expert driver is defined as who can control the vehicle under a rather high level of HRF for a long duration and will not have the vehicle exceed the handling limit. A driver is considered as reckless if he/she behaves careless and unpredictable during his/her driving tour. Since these driving behaviors cannot be well defined, fuzzy control method is used to identify the four categories of drivers above, as shown in Figure 3. Besides, the author also uses zero-speed-gap velocity $v_{\text {zsg }}$ for steady-state car-following to classify driving behaviors into three types-normal, cautious, and aggressive [23]. Similarly, the drivers are characterized by use of response time and the damping ratio for transient carfollowing, which is treated as a 2 nd order system.

Raz et al. present a system for analyzing and evaluating the performance and attitude of a motor vehicle driver [47]. In this work, factor $g$, on the interval $[0,1]$, represents the weights of available maneuvers (safely executed maneuver and dangerously executed maneuver), and the combination of the two maneuvers is compared against current maneuver to find the closest value of $g$ for reproducing the original maneuver, as shown in Figure 4. Thus, $g$ represents the

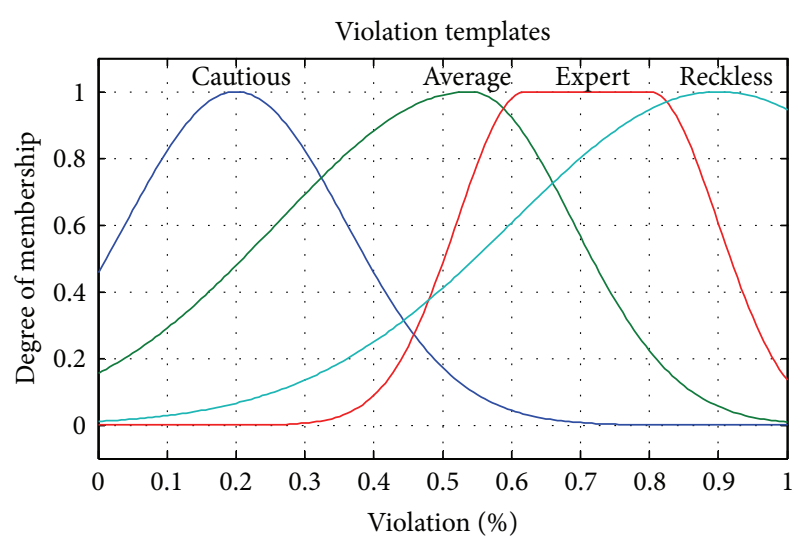

FIGURE 3: Membership functions characterizing the four driver categories based on the handling risk factor, adopted from [12].

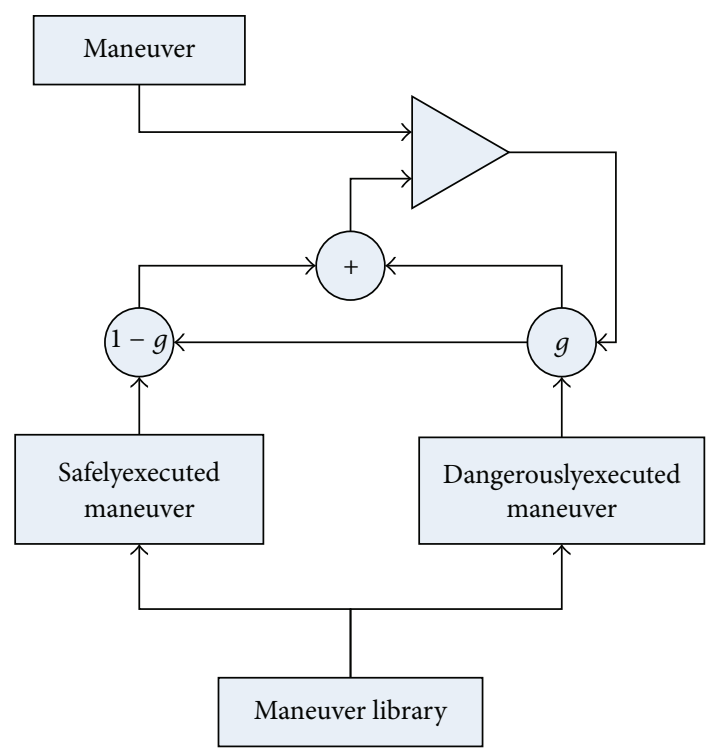

FIGURE 4: A conceptual block diagram of an arrangement for assessing driver attitude, adopted from [47].

driver's attitude for the current maneuver, and fuzzy logic method combined with statistical method is used to classify the driving maneuvers, in terms of the value of $g$. In $[54,55]$, a consolidated fuzzy clustering algorithm is developed and implemented to classify different car-following conditions including stable following, acceleration, approaching, braking, and opening using the pretreated data.

A common disadvantage of fuzzy-algorithm-based is that the thresholds are solely defined by the a priori knowledge of modelers, possibly with bias. A consolidated method, which can calibrate some psychological thresholds based on properties in real data, has not been developed ever [56]. One possible approach to solving the above-mentioned problem is to use supervised classification methods [57], for example, Bayesian classification, but it requires detailed a priori knowledge (e.g., probability distributions of certain variables) in different maneuvers. 


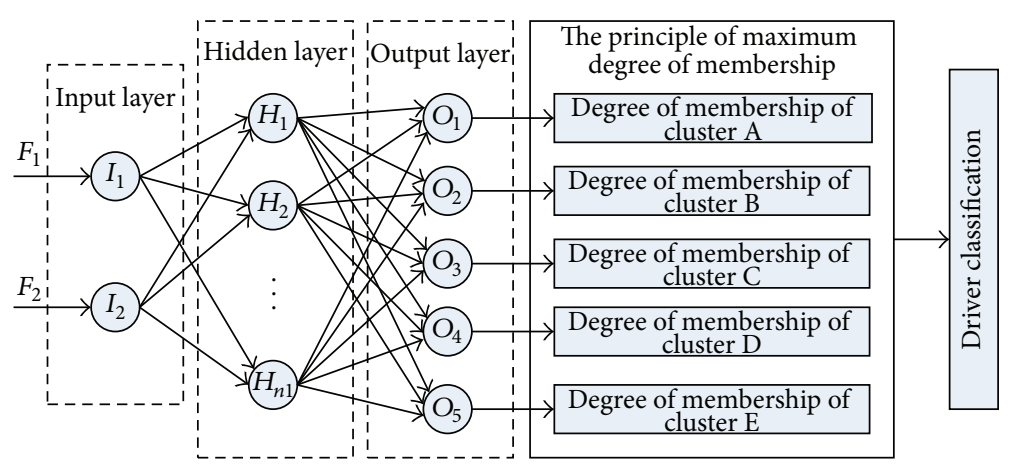

FIgURE 5: The structure of driver classifier based on Back Propagation neural network (BPNN), adopted from [35].

K-means clustering algorithm, also known as ISODATA (Iterative Self-Organizing Data Analysis Techniques Algorithm) [58], is a widely used unsupervised clustering algorithm, which can classify multidimensional data into different groups on the basis of certain dissimilarity measures. Wang et al. use the efficient K-means clustering algorithm to classify the determinants of longitudinal driving behavior, which is acquired from 11 systems and control-related parameters, with the indicated opposite extreme values: aggressive versus prudent, unstable versus stable, risk prone versus risk infrequent, nonskillful versus skillful [33]. Specifically, according to the data sequence with car-following condition, the time to collision (TTC) data of the driver releasing the accelerator pedal and starting braking are extracted and utilized to classify drivers into three categories by clustering analysis method, namely, cautious, normal and aggressive [34].

Besides, driver classifier is designed by Zhang based on neural network [35]. In this work, the author presents that driver behavior inputs are obtained through the humanmachine interface, and the system automatically classifies the driver to achieve self-learning and the parameters automatically match for driver's abnormal behavior characteristic, as shown in Figure 5. In addition, Quintero et al. propose the driving behaviors classifier based on the existing intelligent driving diagnosis system, classifying drivers into two types, aggressive and moderate [8]. Furthermore, Ishibashi et al. develop "Driving Style Questionnaire" (DSQ) to characterize drivers [59].

\subsection{Methods of Building Identification Models of Driver} Behavior Characteristics. Identification of driver behavior characteristics is a pattern recognition process. Since driver behavior characteristics differ in different road surfaces, driving maneuvers, driver profiles, and vehicle dynamics [60], some requirements to the selected modeling methods are necessary: (1) offer a robust processing, that is, with the abilities to detect, approximate, and classify, and with a high reject ratio for the noise and (2) work based on learned cases. The existing attempts to model the driver behavior characteristics are dominated by models that are inspired by neural network (NN) $[8,36,45,61-64]$, Hidden Markov Model (HMM) [17, 24, 25, 38-41, 65-67], fuzzy control theory
[12, 18, 23, 68, 69], Gaussian Mixture Model (GMM) [28$32,70]$, and other models [17, 21,71-74]. In the following paragraphs, the work exemplified will be reviewed in some detail.

3.2.1. Neural Network (NN) Model. The motivation of using an NN approach to behavior identification stems from the desire to conduct efficient searches of various types of driving behaviors located within relatively large amounts of stored time history data. The precision of feature parameters is crucial to the accuracy of NN. If feature parameters of different types are similar or overlapped, the model may not be capable of obtaining demanded accuracy. The pattern recognition ability of certain $\mathrm{NN}$ architectures is well known and lends itself well to this type of task [8, 36, 45, 61-64].

Through implementing and testing two artificial neural network (ANN) topologies: Back Propagation (BP) and Learning Vector Quantization (LVQ) [8], Quintero et al. take advantage of BP to build driver identification model. Besides, the author finds that topology of feed-forward neural network (FFNN) algorithms trained with BP is expert in designing intelligent diagnostic systems and is able to offer a strong learning ability, even with considerably less training samples [63]. The cerebellar model articulation controller (CMAC), developed by Albus, is one of NN architecture and has the advantages of fast learning and a high convergence rate [64]. Thus Wahab et al. propose the use of CMAC to model each driver's behaviors [45].

3.2.2. Hidden Markov Model (HMM). HMM is fundamentally a statistical model. Since the construction of such a model involves assuming a Markov process, it has the ability to determine the hidden states from the observable states of certain systems [75]. An HMM is capable of capturing the dynamic movement of a time series (series arrayed in chronological order), and the states of HMM can be hierarchically organized to describe both short-term and long-term driving behaviors. For example, in the case of driving a vehicle, the long-term driving behaviors represent driving intentions (e.g., accelerating/turning/following/changing lane), while the short-term driving behaviors represent driver's operation behaviors, for example, hitting the steering wheel and pressing the gas pedal [24]. 
TABLE 1: Rules for driving behavior characterization.

\begin{tabular}{|c|c|c|c|}
\hline $\begin{array}{l}\text { If gap time } \\
\text { is }\end{array}$ & $\begin{array}{l}\text { If accelerator } \\
\text { pedal rate STD } \\
\text { is }\end{array}$ & $\begin{array}{c}\text { If brake } \\
\text { pedal rate STD } \\
\text { is }\end{array}$ & $\begin{array}{l}\text { Then driver } \\
\text { index is }\end{array}$ \\
\hline Low & Low & Low & $\begin{array}{c}\text { Less } \\
\text { aggressive }\end{array}$ \\
\hline High & Low & Low & Cautious \\
\hline Low & High & Low & Aggressive \\
\hline Low & Low & High & Aggressive \\
\hline Low & High & High & Aggressive \\
\hline High & High & High & $\begin{array}{c}\text { Less } \\
\text { aggressive }\end{array}$ \\
\hline High & Low & High & Cautious \\
\hline High & High & Low & $\begin{array}{c}\text { Less } \\
\text { aggressive }\end{array}$ \\
\hline
\end{tabular}

Takano et al. propose a hierarchical model with one HMM characterizing the short-term driving behaviors in the lower layer, and the other HMM characterizing the longterm driving behaviors which are represented in the HMM space [25]. This structure makes the vehicles intelligent by storing the knowledge of driving behaviors as the symbols of driving intention through observing the driving behavior given by expert drivers. Baum-Welch algorithm (a maximum likelihood estimation method) which trains parameters of HMMs is applied to optimize three HMMs - driving straight, normal steering, and emergency steering $[38,39]$. In addition, the model [40] based on the combination of HMM and NN model is presented, which can achieve the driving intention recognition and the driving behavior prediction. In [67], driver behaviors are modeled by using HMM in two alternative ways. Using the measured data of driving behaviors, an HMM consisting of three recognition categories-emergency lane change (LCE), ordinary lane change (LCN), and lane keeping (LKN) - is developed [17].

3.2.3. Fuzzy Control Theory. For the pattern recognition systems whose parameters' range is difficult to determine but can be divided according to the a priori knowledge or commonsense, fuzzy control theory is available to model it.

Lu et al. [12] use fuzzy subsets to category drivers by introducing the HRF (see Figure 3). First degree of membership is calculated to each of the four categories (i.e., cautious, average, expert, and reckless) for each event of a specific driver; then a probabilistic method is used to calculate the possibilities that are generated by multiple events and to aggregate the overall possibilities in order to characterize the driver.

$\mathrm{Lu}$ also proposes a driver-in-the-loop system (see Figure 6) and uses three methods to characterize driver's driving behaviors or control structure in real time. Table 1 illustrates fuzzy rules of a Takagi-Sugeno model to realize semistructured driving behavior characterization. In addition, an evolving Takagi-Sugeno fuzzy model is presented for capturing the evolving characteristics of driving behaviors [23].

3.2.4. Gaussian Mixture Model (GMM). GMM is a parametric approach to density estimation [76]. GMM is known for its ability to generate arbitrarily shaped densities, and it has experienced extensive use in pattern recognition, such as speech recognition and speaker recognition. Miyajima et al. has been working on modeling driving behavior based on GMM and written several related papers [27-32].

In [29], GMM is applied to identify drivers in the case of car-following condition, with the accuracy of $76.8 \%$ by field test data. In [30], driving patterns of each driver are modeled based on GMM. In this work, the GMM is trained as a joint probability distribution of following distance, velocity, pedal position signals, and their dynamics. Experiments conducted using a driving simulator show that car-following conditions reproduced by the GMMs for three different drivers maintain these drivers' individual driving styles.

In addition, by comparing the performance of present driver-behavior models for car-following condition based on GMM and based on piecewise auto regressive exogenous (PWARX) algorithms, Miyajima et al. find that the PWARXbased model takes slightly advantage over the GMM-based model for all cases [29]. Furthermore, the literature [31] certifies that the GMM-based model performs better when the measured parameters of diving behaviors are used, but under such circumstance the model becomes more sensitive to the approximation errors of the input parameters as in the recursive prediction. The literature also confirms that the PWARX-based model performs better than the GMMbased model at the long-term prediction but not at the short-term prediction. This is because of the feature of the PWARX; namely, it captures the relationship among the driving behaviors at long-time duration, finally makes the PWARX-based model more generalized, and sets abound to the prediction errors.

3.2.5. Other Models. Apart from the aforementioned methods, there are other ways to represent a driver's behavior. Bifulco et al. analyze and compare the performance of linear, polynomial, and FFNN approach to model driving behaviors, proposes that a linear model is not actually overperformed by more complicated approaches and that it is worth adopting in light of its great simplicity [72, 73]. Adaptive Network based Fuzzy Inference System (ANFIS) [74] draws the predominance of NN and fuzzy control efficiently and has high learning efficiency, high training efficiency, and good network generalization capability. The hybrid driver model [71] is able to reproduce the driving behaviors of different driver characteristics, so it can adapt to various types of drivers (e.g., good, novice, and fast).

3.2.6. Summary. It is increasingly recognized that intelligent and personalized vehicle systems are developed based on certain intelligent algorithms to which have a knowledge base. Such systems could process the received sensory data not only quantitatively but also qualitatively, for example, 


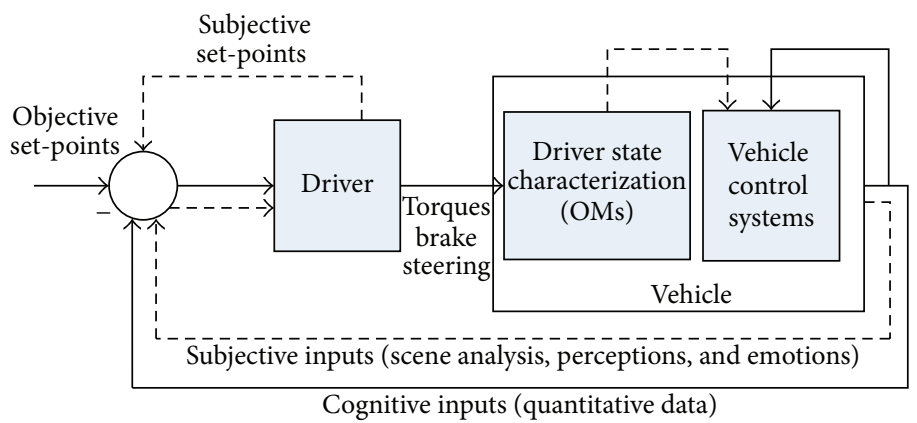

FIGURE 6: Cognitive (solid line) and subjective (broken line) flow of information in a driver-vehicle system, adopted from [18].

interpret the driving behavior data, then compare it with the stored data in the behavior base, in some case add this new driving behavior data to the behavior base, and finally identify the driver behavior characteristics. Several methods of building identification models of the driver behavior characteristics have been introduced above. Each method has its own pros and cons, as shown in Table 2.

3.3. Experimental Design and Data Acquisition. In general, during the design of experiment, it is necessary to consider experimental participants, experimental vehicles, test maneuvers, and data acquisition. In particular, for building identification models of the driver behavior characteristics, the above aspects should be carefully determined.

3.3.1. Experimental Participants. First, experimental participants must have their own driving licenses. In addition, the number of the selected drivers should be large enough to cover all possible drivers' characteristics researched. For example, four driver characteristics-cautious, average, expert, and reckless-are described in [12], so the drivers with these driving styles should be chosen as experimental participants. It should be noted that even for the same driver, driver characteristic may alter from situation to situation, for example, fatigue, drunk, and drowsy. Therefore, experiment participants should participate in experiments under not overly tension and fatigue status.

3.3.2. Experimental Test. In order to obtain a diverse range of driver characteristics, a large number of experiments on vehicles with different dynamics and on different roads should be conducted. This is believed to be one of the reasons that the driving simulator experiment is used instead of field test. In [8], the Racer Simulator (created by Ruud van Gaal) is used to conduct experiments in Figure 7. Other relevant reviews include $[79,80]$.

In addition to the driving simulator, the instrumented vehicles have been developed to conduct experiments [67, $70,81]$. The UTDrive Vehicle converted from a Toyota RAV4 (see Figure 8) is used to collect data from real-road experiments. Additionally, a data collection vehicle, which is called TOYOTA REGIUS [81], has been specially designed

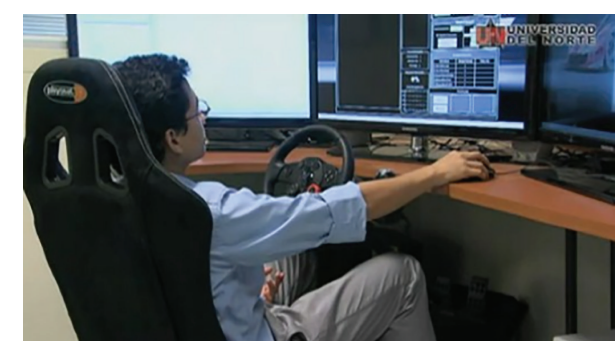

FIGURE 7: Driving platform for simulated experiments, adopted from $[8]$.

for data collection in the Center for Integrated Acoustic Information Research (CIAIR) project.

3.3.3. Test Maneuvers. Driver behavior characteristics can be subdivided into steering characteristic, acceleration characteristic, and braking characteristic. In order to have full access to the driver behavior characteristics, it is necessary to design experiments with all test maneuvers in connection with certain characteristic. For the steering characteristic, the test track should be designed to one with a variety of bend radiuses, as shown in Figure 9. For the acceleration characteristic and the braking characteristic, most existing references $[18,29,30,82,83]$ are available with optional carfollowing maneuvers.

Especially, when conducting driving simulator experiments, using computer-generated time history curve of certain variables about test maneuvers to design experiments, is easier to achieve all test maneuvers. Figure 10 gives two velocity patterns of the lead vehicle which are used. The upper velocity pattern is recorded in an express way driving scenario in the driving simulator, and the lower pattern is an artificial velocity pattern by use of software aimed at obtaining all velocity ranges. Obviously, the test maneuver generated by computer is more comprehensive than that by driving simulator.

\subsubsection{Data Acquisition}

(1) Feature Parameters Chosen. Since identification of driver behavior characteristics is a pattern recognition process, it can be modeled based on pattern recognition methods by 
TABLE 2: Features of the four methods of building identification models.

\begin{tabular}{|c|c|c|c|c|}
\hline $\begin{array}{l}\text { Identification } \\
\text { methods }\end{array}$ & $\mathrm{NN}$ & HMM & Fuzzy control theory & GMM \\
\hline Algorithm features & $\begin{array}{l}\text { To identify various types of } \\
\text { driving behaviors located } \\
\text { within relatively large } \\
\text { amounts of stored time } \\
\text { history data. The quality of } \\
\text { feature parameters is } \\
\text { crucial to the accuracy of } \\
\text { NN. }\end{array}$ & $\begin{array}{l}\text { To describe the statistical } \\
\text { properties of stochastic } \\
\text { processes and to identify } \\
\text { inherent invisible states } \\
\text { through external } \\
\text { observation sequence. }\end{array}$ & $\begin{array}{l}\text { To formulate fuzzy rules } \\
\text { based on previous } \\
\text { experience and then design } \\
\text { model performance in } \\
\text { accordance with the } \\
\text { expectations of the } \\
\text { designer. }\end{array}$ & $\begin{array}{l}\text { A parametric approach to } \\
\text { density estimation is able to } \\
\text { generate arbitrarily shaped } \\
\text { densities. }\end{array}$ \\
\hline Model accuracy & Very high & Very high & High & High \\
\hline $\begin{array}{l}\text { Real-time } \\
\text { performance }\end{array}$ & Fair [37] & Very good [37] & Fair [77] & $\begin{array}{l}\text { The traditional GMM is } \\
\text { poor, and the advanced } \\
\text { GMM is good [78]. }\end{array}$ \\
\hline Model adaptive & - & - & - & $\begin{array}{l}\text { Using the maximum a } \\
\text { posteriori (MAP) or } \\
\text { Bayesian adaptive } \\
\text { algorithm to adjust } \\
\text { parameters of GMM, } \\
\text { personalized driver } \\
\text { behavior model will be } \\
\text { obtained. }\end{array}$ \\
\hline Disadvantages & $\begin{array}{l}\text { There is not a unified } \\
\text { feasible method to adjust } \\
\text { parameters (e.g., the } \\
\text { number of NN layers) but } \\
\text { generally subjective } \\
\text { adjustments based on the } \\
\text { simulation results of the } \\
\text { models; training time is } \\
\text { long. }\end{array}$ & $\begin{array}{l}\text { HMM is not suitable for } \\
\text { long-term forecasting } \\
\text { system and requires } \\
\text { artificial hypothesis for the } \\
\text { sequence distribution of the } \\
\text { current states. }\end{array}$ & $\begin{array}{l}\text { Since its fuzzy rules are } \\
\text { formulated based on a } \\
\text { priori knowledge, the } \\
\text { simulation results may } \\
\text { deviate from the actual } \\
\text { values. }\end{array}$ & $\begin{array}{l}\text { GMM cannot obtain more } \\
\text { efficient modeling of the } \\
\text { time series of feature } \\
\text { vectors than other methods } \\
\text { do. }\end{array}$ \\
\hline Applications & $\begin{array}{l}\mathrm{NN} \text { is suitable for pattern } \\
\text { recognition that is easy to } \\
\text { access to acquire the feature } \\
\text { parameters, such as music } \\
\text { recognition and speech } \\
\text { recognition. }\end{array}$ & $\begin{array}{l}\text { HMM is suitable for } \\
\text { pattern recognition with } \\
\text { strong time series data, } \\
\text { such as driver's intention } \\
\text { recognition and speech } \\
\text { recognition. }\end{array}$ & $\begin{array}{l}\text { Fuzzy control theory is } \\
\text { suitable for pattern } \\
\text { recognition whose } \\
\text { parameter range is difficult } \\
\text { to determine. }\end{array}$ & $\begin{array}{l}\text { GMM is expert in } \\
\text { identifying short-term } \\
\text { driving behaviors but not in } \\
\text { long-term driving } \\
\text { behaviors. If combined } \\
\text { with PWARX, the model } \\
\text { can have a good } \\
\text { performance both in the } \\
\text { short- and long-term } \\
\text { driving behaviors. }\end{array}$ \\
\hline
\end{tabular}

using feature parameters of the drivers' driving behaviors. Therefore, the parameters must be selected in a way that it is relevant to a human driver's characteristics, and the number of feature parameters is crucial to the accuracy of the identification models of the driver behavior characteristics. Particularly in practice when the amount of individual driving data used to establish the identification models is relatively smaller than that of the development data, the models obtained from such sparse data may not be able to represent the driver behavior characteristics in a typical manner [27]. In general, the data includes driver's maneuvers and vehicle states information, such as vehicle speed, yaw rate, lateral and longitudinal accelerations, steering angle, steering angle velocity, brake pedal position and its derivative, acceleration pedal position and its derivative, and others relative to a certain test maneuver.
Through a large number of tests and analysis, the rules selecting feature parameters are summarized. Wakita et al. [84] find that the nonparametric models take advantage over the parametric models and explains that the driver's operation behaviors are better than both adhesion conditions and vehicle states information. According to previous work [85], the gas and brake pedal signals chosen as the modeling data are adopted to build identification model, with best computational efficiency and high identification accuracy. However, the experimental results also show higher accuracy of driver identification when the modeling data is combined with the derivative of the gas and brake pedal signals (e.g., rate of the gas pedal), instead of the original gas and brake pedal signals collected [45]. Compared with raw driving signals, frequency responses calculated by spectral analysis of driving behaviors could better capture the individualities in driving 


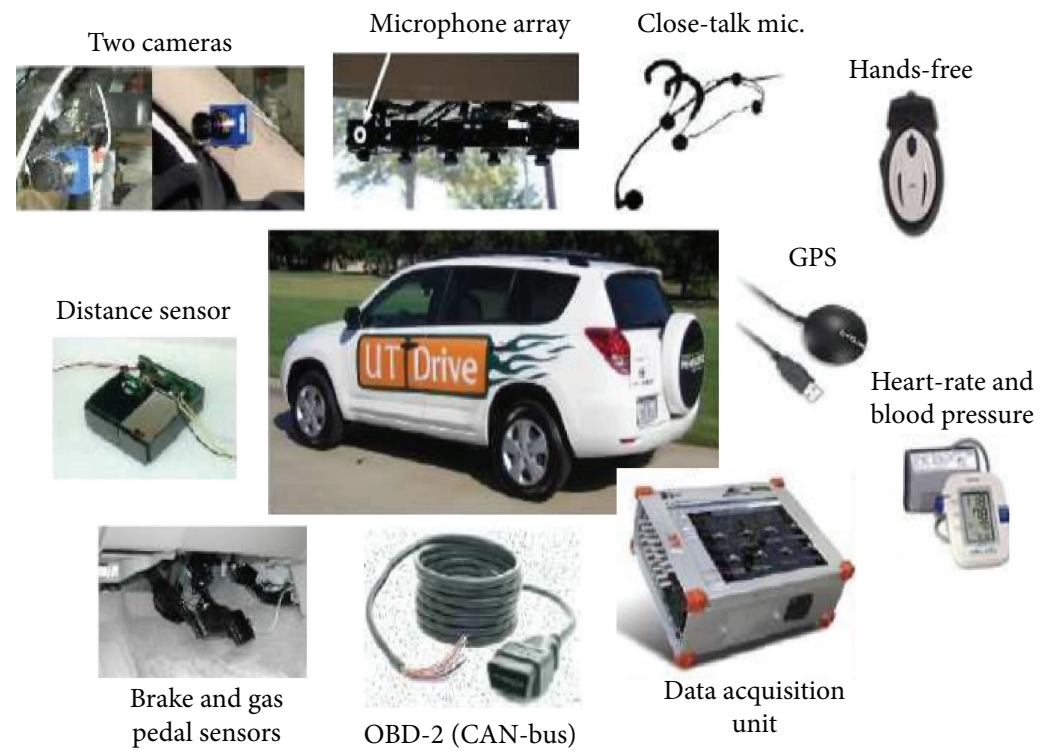

FIGURE 8: UTDrive data collection vehicle, adopted from [67].

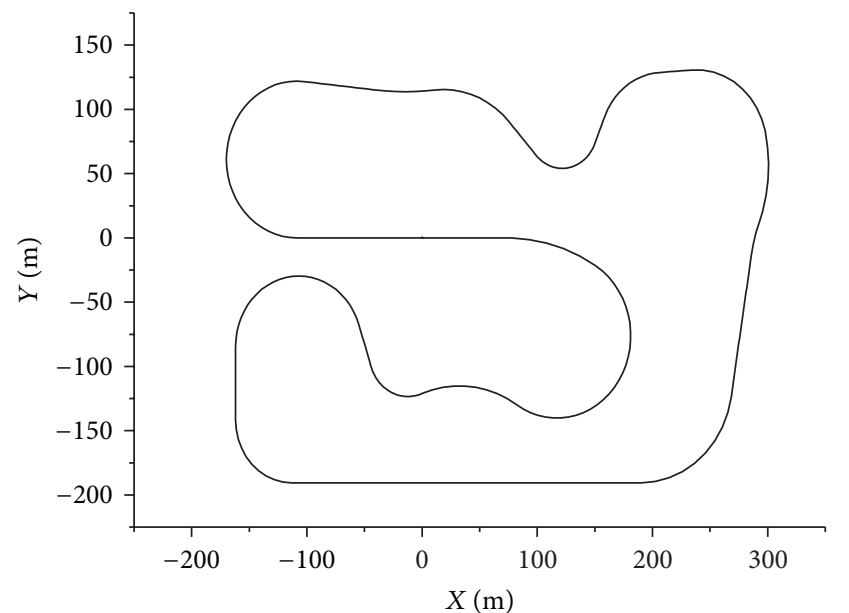

FIGURE 9: The test track for the steering characteristic, derived from the test maneuvers of CarSim.

behaviors and could receive better performance in identifying the drivers [81].

(2) Data Processing. Data would generally be filtered, converted, grouped, and so forth. In addition, different pattern recognition methods have different requirements to data. With regard to $\mathrm{NN}$, in order to build the intelligent driver behavior models and to learn different drivers' characteristics, input data to NN should be representative parameters of driver behaviors under different maneuvers. The following statistical measures of central tendency may be used: range, media, standard deviation, variance, and mean absolute deviation [8]. While for PWARX model, the input and output parameters are first clustered and categorized into different driving modes [31].
3.4. Model Adaptation. The identification models of driver behavior characteristics mentioned above are static models. Overall, the prediction performance of these models decreases as the driving maneuvers change from the simulation scenarios with controlled environment to the real-world driving environments under diverse uncontrolled factors [27]. In the real-world driving environments, the profiles and driving intentions are unknown; the driver may not behave exactly as the identification models represent, which results in bias of the models. In addition, the inaccuracy of signals collected from the sensory systems should be taken into account. Therefore, to solve these problems and to develop a more reliable model, model adaptation has been presented and regarded as one of the foremost solutions.

In general, the main objectives of model adaptation are to cope with (1) relatively small amount of observed driving data of individual driver, (2) individual driving style or characteristic that differs from the designed average style, and (3) mismatch of driving maneuvers between development and usage stages.

Angkititrakul et al. propose a method to execute drivermodel adaptation [31]. The universal driver-behavior models are first built by use of driving data of several drivers based on certain identification method (e.g., HMM). The universal driver-behavior models represent average/common driver characteristics shared by these drivers. The author takes further measures to adjust the parameters of the universal driver-behavior models in the following two scenarios: (1) driver adaptation: the driving data of each driver are used to adjust the universal driver-behavior model to build the adapted driver models, namely, driver-dependent or personalized driver models. (2) On-line adaptation: the driving data at the beginning of each driving event (e.g., car-following condition) are used to adjust the universal driver-behavior model and subsequently the on-line adapted driver model 


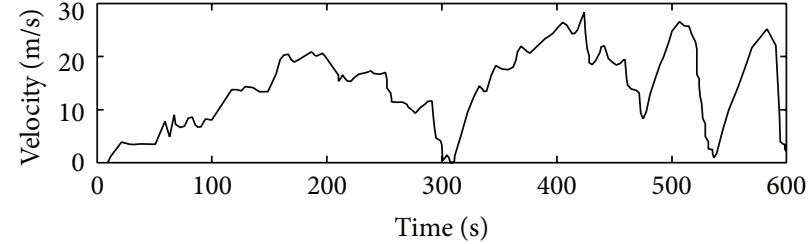

(a)

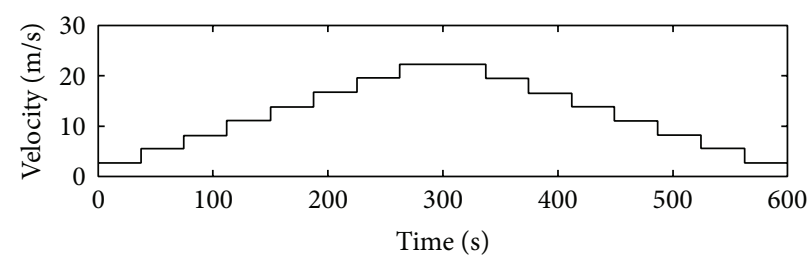

(b)

FIGURE 10: Velocity patterns of lead vehicle, adopted from [30].

is utilized to represent driving behaviors for the rest of that driving event.

\section{Applications in Identification of Driver Behavior Characteristics for Automotive Control}

4.1. Intelligent Driver Advisory System. The main objective of the state-of-the-art vehicle electronic control systems is to improve vehicle performance by identifying driver intentions/characteristics and controlling the vehicle to realize the driving intentions safely, robustly, and smoothly. The performance of electronic control systems can be significantly improved when the driver and the electronic control systems could cooperate with each other to pursue the same hazard avoidance goal and to maximize the accident avoidance capability of the driver-in-the-loop vehicle as a system.

Lu develops a driver advisory system based on the driver characteristics that can warn the drivers of driving conditions when the vehicle approaches to its limit, which is a part of a cluster of warning functions defined as an Intelligent Personal Minder (IPM) system. Figure 11 depicts the interaction of the IPM system with the other subsystems and functions. The electronic control system follows the driver intentions and the driver responds to the advisory information from the electronic control system to modify his/her operation inputs (e.g., rising braking pedal, increasing steering wheel angle, etc.). In this way a seamless coordination between the driver and the electronic control system could be realized and it is likely to minimize the effect of the potential safety hazards resulted from driver's operation errors.

4.2. Driver Safety Warning System. Introducing the driver behavior characteristics to design the driver safety warning system, the redundancy alarm rate can be reduced and the negative interference on the driver will be improved. Wang et al. present vehicle collision warning/avoidance algorithms based on the driver behavior characteristics $[34,86]$. By changing parameters, the algorithms can better adapt to the behavior of different drivers. Take the longitudinal driving maneuver for example, the different level safe thresholds, warning rules, and warning logic are determined using time headway (THW) and time to collision (TTC).

Kentaro Ogchi et al. invent a system for predicting driver behavior and generating control and/or warning signals. As shown in Figure 12, the driver predictor is comprised of an initialization module, an updating module, a transfer module, a hierarchical temporal memory (HTM), and a prediction retrieval module. The initialization module processes the data from the database and uses it to create initial states or definitions for different driver behaviors in the HTM. After the updating module identifies the driver, the HTM is adjusted to match the driver of the vehicle. The transfer module standardizes the format of real-time data and inputs it to the HTM. In accordance with the information of the initialization module, the updating module and the transfer module, the HTM uses a hierarchical temporal memory construct to predict vehicle states. Then the prediction retrieval module queries the HTM to generate warning control signals to alert the driver of potentially dangerous conditions, to generate collision control signals to prevent or avoid collisions, and to generate acceleration control signals for the adaptive cruise control.

The functional view of the HTM is shown in Figure 13. The HTM consists of long-term storage, intermediate-term storage, short-term storage, an intermediate-to-long-term (ILT) converter and a short-to-intermediate-term (SIT) converter. The HTM has a memory mechanism with a hierarchical structure of temporal memory based on the memory mechanism of the human brain. The information stored in the three storages of the HTM, respectively, represents a current state of the vehicle and other vehicles surrounding it, that is, the short-term storage stores short paths of vehicle action, the intermediate-term storage stores trajectory types, and the long-term storage stores behavior types.

The prediction retrieval module is made up of a storage for driver preferences, a training module, a warning control module, a collision control module, and acceleration control module, as shown in Figure 14. Each of these modules accesses and retrieves data from the HTM. The storage for driver preferences contains sample sets of preferences for different kinds of drivers. Typical preferences stored in the storage for driver preferences include the types and levels of settings for warning control, collision control, and acceleration control. The training module is used to train the warning control module, the collision control module, and the acceleration control module.

4.3. Vehicle Dynamics Control System. X-by-wire control technology comprises steer-by-wire, brake-by-wire, driveby-wire, and suspension-by-wire (active suspensions) subsystems and has higher accurate and more complex control algorithms than traditional automotive control systems do. 


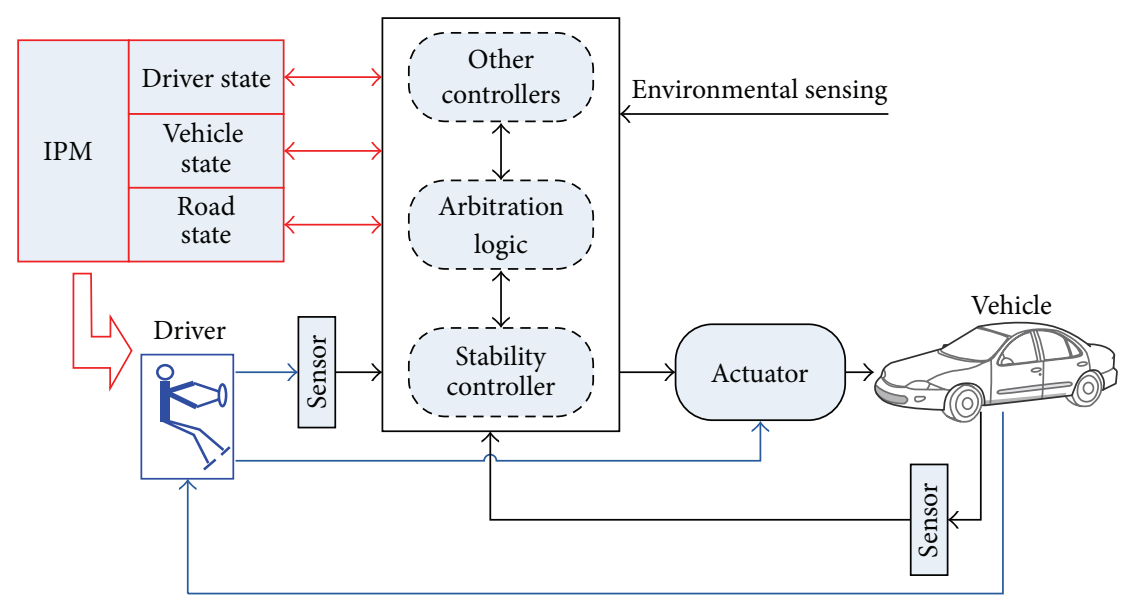

FIGURE 11: The block diagram of a vehicle control system including an IPM, adopted from [12].

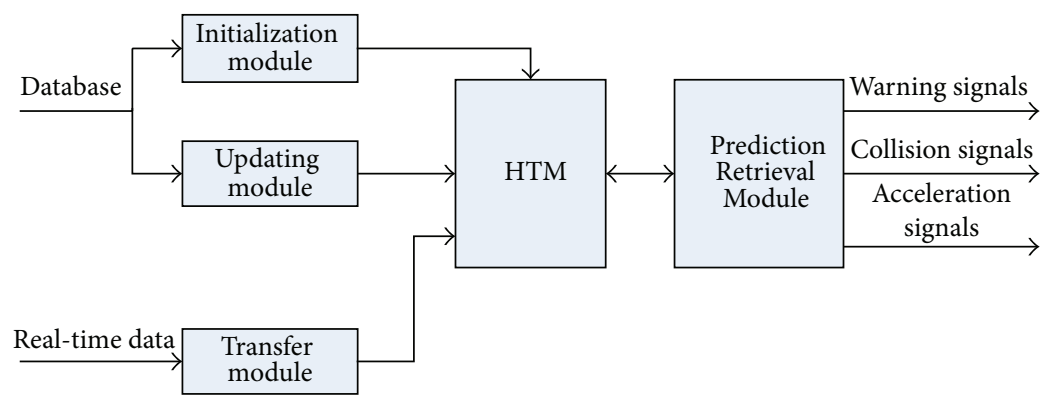

FIGURE 12: A functional view of a driver predictor system, adopted from [21].

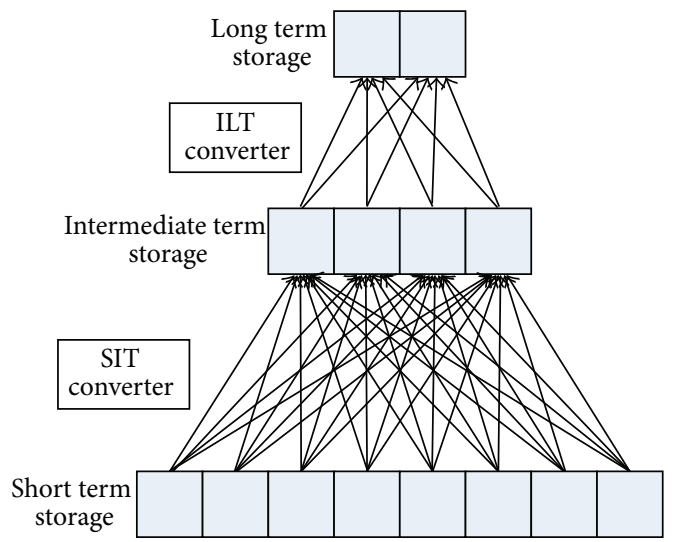

FIgURE 13: A functional view of a hierarchical temporal memory, adopted from [21].

Introducing the intelligent control algorithm to identify driver's intention and driver characteristics, the performance of the driver-vehicle-road closed-loop system will be enhanced, and finally different driving characteristics and personalized ideal vehicle dynamics characteristics will be realized, that is, converting from "driver adapts to car" to "car adapts to driver" $[19,20]$.

The $\mathrm{x}$-by-wire vehicle's controller controls the actuators according to the driver's operation signals, and the vehicle reference models in the controller provide control objectives for the $\mathrm{x}$-by-wire vehicle according to the driver operation signals and vehicle states information. The vehicle reference models play a very important role for controlling the $\mathrm{x}$-bywire vehicle. In order to realize different driving characteristics of the $\mathrm{x}$-by-wire vehicle, we present the control principle of "car adapts to driver" $\mathrm{x}$-by-wire vehicle's ideal dynamics characteristics (see Figure 15). The dotted area is an integrated controller of the $\mathrm{x}$-by-wire vehicle, which exports steering angles, driving, and braking torques of four wheels. The dash dot area shows the identification system of driver (behavior) characteristics. Initially the vehicle uses the original vehicle reference model before the identification system of driver characteristics identifies the driver's characteristics based on the driver's operation signals and vehicle states; thereafter the vehicle reference model is switched to matching characteristic of the ideal reference models, which can control actuators of the $\mathrm{x}$-by-wire vehicle through the integrated controller and the optimal distribution of tire forces, and finally realizes the personalized ideal dynamics outputs for the $\mathrm{x}$-by-wire vehicle.

The identification system of driver characteristics includes the driver characteristics identification models and the ideal reference models. According to driver's operation behaviors, the driver characteristics can be decomposed into steering characteristic, acceleration characteristic, and braking characteristic; correspondingly the identification 


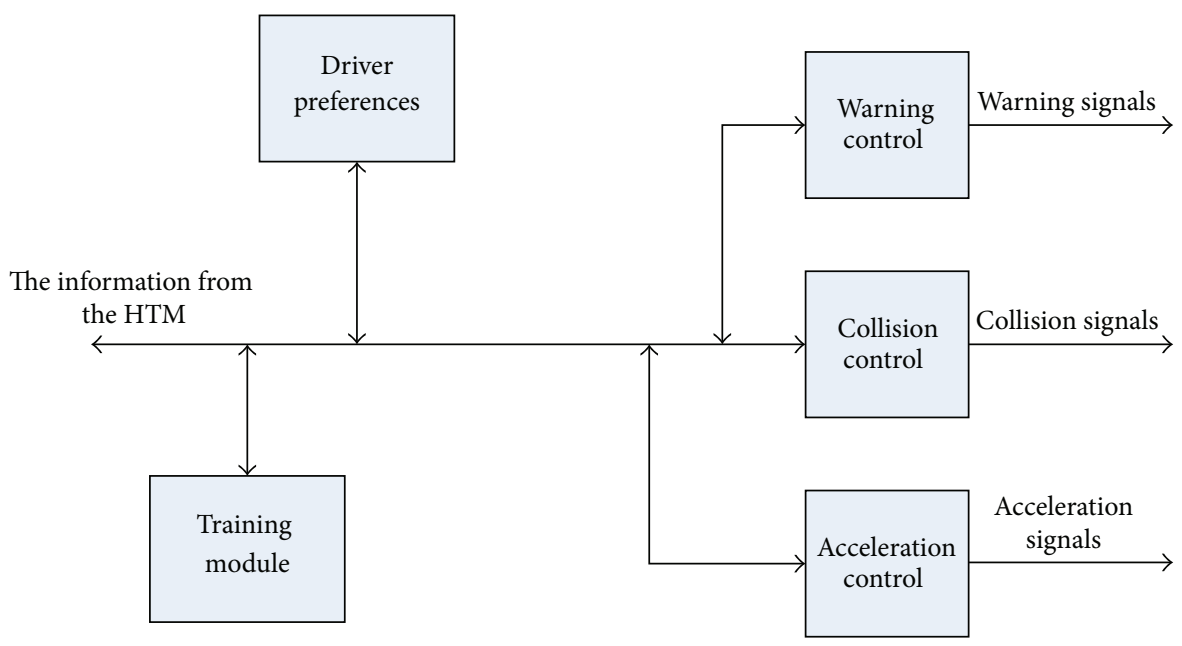

FIGURE 14: A block diagram illustrating a functional view of a prediction retrieval module, adopted from [21].

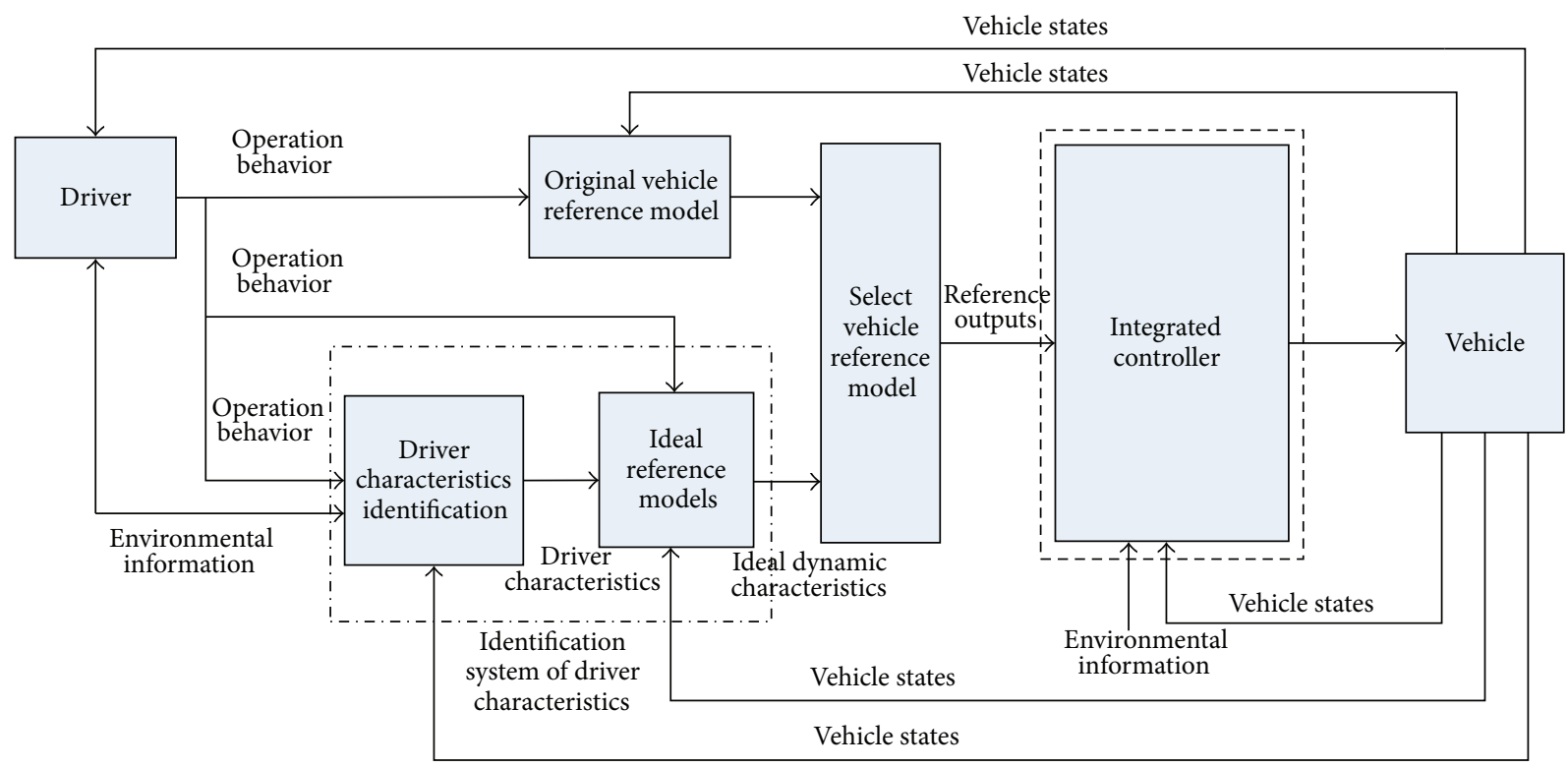

FIGURE 15: Control principle of "car adapts to driver" $\mathrm{x}$-by-wire vehicle's ideal dynamics characteristics.

system is divided into three subsystems, which are the steering module, the acceleration module, and the braking module, as shown in Figure 16. Each module, respectively, identifies the driver's each characteristic; for example, steering characteristic is cautious, acceleration characteristic is average, and braking characteristic is reckless; then the corresponding ideal dynamics reference modelscautious steering, average acceleration, and reckless braking reference model-are automatically matched. Driver's final ideal dynamics reference models consist of the steering, acceleration, and braking models matched and, respectively, provide reference outputs (e.g., yaw rate, sideslip angle, acceleration, and deceleration) to integrated controller for the $\mathrm{x}$-by-wire vehicle. The identification models are built based on NN or HMM considering these two intelligent algorithms' advantages mentioned above.

\section{Conclusion}

Identification and applications of the driver characteristics for automotive control are widely ranging and informative. In the present work, the fundamental of driver behavior characteristics is introduced; the intrinsic link among the driving behavior, the driving intention, and the driver behavior characteristics is explained; the whole process during establishing the identification models of the driver behavior characteristics is summarized and analyzed in detail, including driver characteristics classification, identification methods, experimental design and data acquisition, and model adaptation. On this basis, applications of the driver characteristics for automotive control have been introduced on three aspects, namely, the intelligent driver advisory system, the driver safety warning system, and the vehicle dynamics control system. 


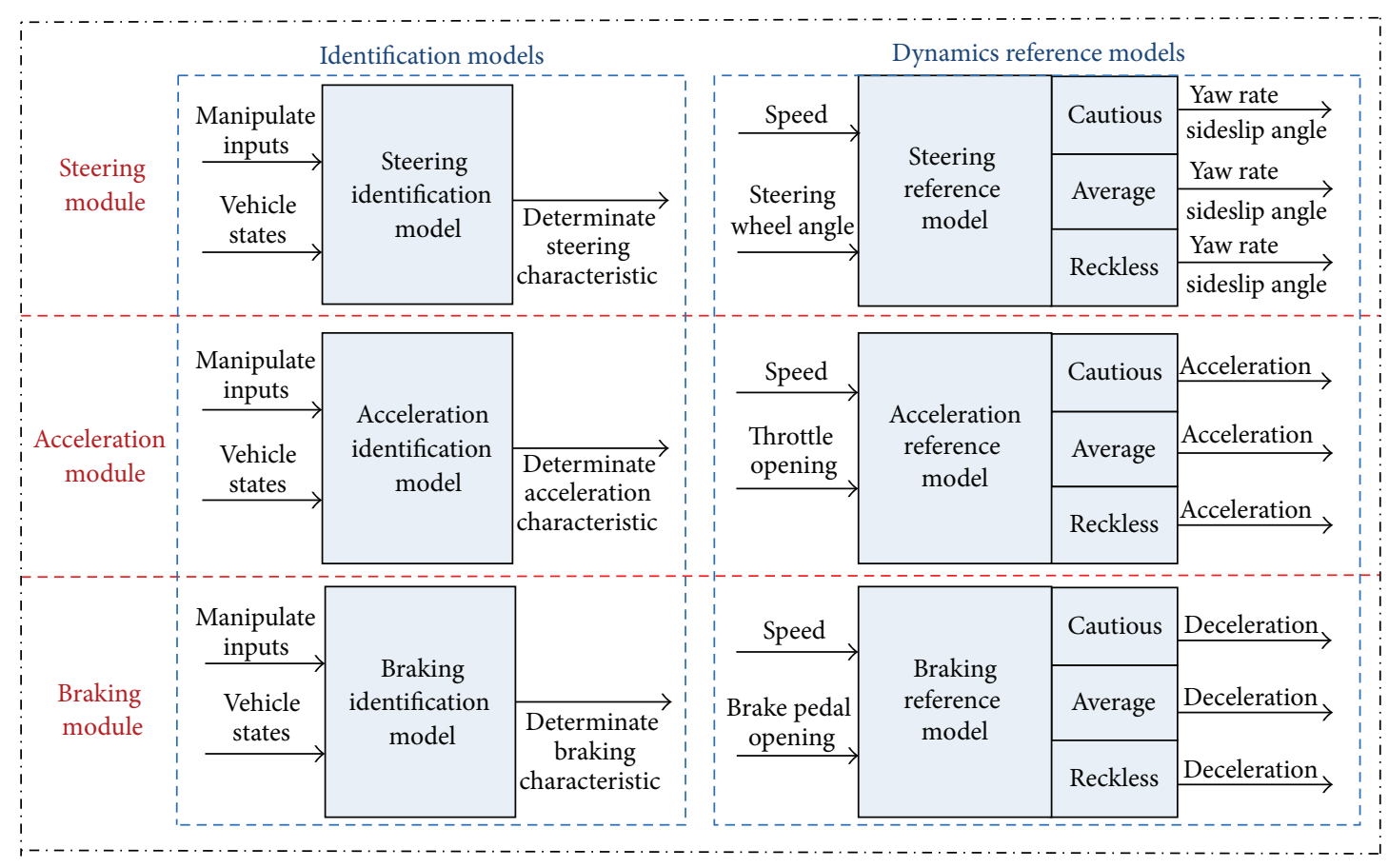

FIGURE 16: Principle of the driver characteristics identification system.

The driver is a complex and uncertain individual, which might exhibit different driving characteristics in different driving situations (fatigue/drunk/drowsy/distracted driving). In addition, different road adhesion, traffic conditions, and weather conditions will also impact driver characteristics. Therefore, extensive experiments are required to be conducted on potential user groups (the groups with the driver characteristics being studied) for the purpose of establishing a comprehensive human driving behavior library for greater precision and wider applications. It will be necessary to ensure robustness of the models in actual driving situations, in addition to improving recognition performance by resolving the aforementioned issues. On-line adaptation of the driver behavior characteristics is considered as one of the foremost solutions. In fact the driver behavior characteristics can be influenced and reaccustomed through learning, so it is of great importance to build online adaptive models of the driver behavior characteristics by using online field test data to revise the parameters of the established models, instead of the static models. In addition, there may exist some cross correlations among the classifications of driver behavior characteristics, which is a topic that deserves further study. Since the existing classifications are very rough, the classification should be further refined, and the ultimate goal is to acquire the driver's personal preference feature.

Driver characteristics have been used to identify driver, to detect driver's abnormal behaviors, to design driver assistance systems which adapt to individual driver, to establish different types of driver models to intelligently assist individual driver, and so forth. Designing ideal dynamics reference models adaptation to driver characteristics for the $\mathrm{x}$-by-wire vehicles, then making it realize that "driver adapts to car" changes to "car adapts to driver", and finally achieving personalized driving are a very interesting and promising application of the driver characteristics for automotive control. With further research, applications of the driver characteristics will be broadened in the future.

\section{Conflict of Interests}

The authors declare that they have no conflict of interests regarding the publication of this paper.

\section{Acknowledgments}

This work was supported by the Proposal of the Open Fund Project of the State Key Laboratory of Automotive Simulation and Control under Grant no. 20120111 and by the National Youth Natural Science Foundation of China under Grant no. 51305190 .

\section{References}

[1] W. Rongben, G. Lie, T. Bingliang, and J. Lisheng, "Monitoring mouth movement for driver fatigue or distraction with one camera," in Proceedings of the 7th International IEEE Conference on Intelligent Transportation Systems (ITSC '04), pp. 314-319, October 2004.

[2] L. M. Bergasa, J. Nuevo, M. A. Sotelo, R. Barea, and M. E. Lopez, "Real-time system for monitoring driver vigilance," IEEE Transactions on Intelligent Transportation Systems, vol. 7, no. 1, pp. 63-77, 2006.

[3] Z. Tao, The study of fatigue detection system of drivers based on the eye status [Ph.D. thesis], Zhejiang Sci-Tech University, 2012. 
[4] W. El Falou, J. Duchêne, M. Grabisch, D. Hewsona, Y. Langerona, and F. Linoc, "Evaluation of driver discomfort during long-duration car driving," Applied Ergonomics, vol. 34, no. 3, pp. 249-255, 2003.

[5] S. J. Park and H. K. Lim, "Characteristics of elderly driver's driving behavior and cognition under unexpected event using driving simulator," SAE 2011-01-0552, 2011.

[6] X. H. Zhao, R. X. Fang, J. Rong et al., "Experiment study on comprehensive evaluation method of driving fatigue based on physiological signals," Journal of Beijing University of Technology, vol. 37, no. 10, 2011.

[7] T. Bellet, P. Mayenobe, J. C. Bornard, J. C. Paris, D. Gruyer, and B. Claverie, "Human driver modelling and simulation into a virtual road environment," in Human Modelling in Assisted Transportation: Models, Tools and Risk Methods, P. C. Cacciabue, M. Hjälmdahl, A. Lüdtke, and C. Riccioli, Eds., pp. 251-262, Springer, Milan, Italy, 2011.

[8] M. Quintero, G. Christian, J. O. Lopez, and A. C. C. Pinilla, "Driver behavior classification model based on an intelligent driving diagnosis system," in Proceedings of the 15th International IEEE Conference on Intelligent Transportation Systems (ITSC '12), pp. 894-899, Anchorage, Alaska, USA, September 2012.

[9] H. Yoshida, M. Nagai, T. Kamada, and Y. Shimozato, "Experiment and design of advanced brake assist system for active interface vehicle based on driver's gas pedal operation," JSAE Review, vol. 26, no. 2, pp. 237-243, 2005.

[10] R. Chi, J. Wang, F. Yang, and L. Zhang, "A forward collision warning algorithm based on driver characteristics," in Proceedings of the 10th International Conference of Chinese Transportation Professionals (ICCTP '10), pp. 640-652, 2010.

[11] M. Ishibashi, M. Okuwa, and M. Akamatsu, "Driver's characteristics index of driving style/workload sensitivity for a driving behavior study," Journal of the Society of Automotive Engineers of Japan, vol. 58, no. 12, pp. 34-39, 2004.

[12] J. Lu, D. Filev, K. P. Asante, F. Tseng, and I. V. Kolmanovsky, "From vehicle stability control to intelligent personal minder: real-time vehicle handling limit warning and driver style characterization," in Proceedings of the IEEE Workshop on Computational Intelligence in Vehicles and Vehicular Systems (CIVVS '09), pp. 43-50, Nashville, Tenn, USA, April 2009.

[13] Jianbo Lu, "Modern vehicle dynamics and controls for automotive adaptation, personalization and safety," in Proceedings of the International Forum on Advanced Vehicle Technologies and Integration (VTI '11), Changchun, China, 2011.

[14] G. Xi and Y. Qun, "Driver-vehicle-environment closed-loop simulation of handling and stability using fuzzy control theory," Vehicle System Dynamics, vol. 23, supplement 1, pp. 172-181, 1994.

[15] A. Y. Ungoren and H. Peng, "An adaptive lateral preview driver model," Vehicle System Dynamics, vol. 43, no. 4, pp. 245-259, 2005.

[16] M. Fujisawa, T. Hasegawa, T. Ishii, M. Sakamoto, and S. Sasaki, "Apparatus and method for automobile control using a control characteristic which can be adjusted by the driver," U.S. Patent 5467277, 1995.

[17] N. Kuge, T. Yamamura, O. Shimoyama, and A. Liu, "A driver behavior recognition method based on a driver model framework," SAE 2000-01-0349, 2000.

[18] D. Filev, J. Lu, K. Prakah-Asante, and F. Tseng, "Real-time driving behavior identification based on driver-in-the-loop vehicle dynamics and control," in Proceedings of the IEEE International Conference on Systems, Man and Cybernetics (SMC '09), pp. 2020-2025, San Antonio, Tex, USA, October 2009.

[19] http://msc.berkeley.edu/research/automotive/ev.html.

[20] C.-F. Zong, N. Lin, G. Li et al., "Neural network modeling of ideal characteristics reference model for "car adapts to driver" X-by-wire vehicle," Journal of Jilin University: Engineering and Technology Edition, vol. 43, pp. 514-520, 2013.

[21] K. Oguchi and D. Weir, "System for predicting driver behavior," U.S. Patent 11/968, 864, 2008.

[22] J. Yi, J. Li, J. Lu, and Z. Liu, "On the stability and agility of aggressive vehicle maneuvers: a pendulum-turn maneuver example," IEEE Transactions on Control Systems Technology, vol. 20, no. 3, pp. 663-676, 2012.

[23] D. Filev, J. Lu, F. Tseng, and K. Prakah-Asante, "Real-time driver characterization during car following using stochastic evolving models," in Proceedings of the IEEE International Conference on Systems, Man, and Cybernetics (SMC '11), pp. 1031-1036, Anchorage, Alaska, USA, October 2011.

[24] A. Pentland and A. Liu, "Modeling and prediction of human behavior," Neural Computation, vol. 11, no. 1, pp. 229-242, 1999.

[25] W. Takano, A. Matsushita, K. Iwao, and Y. Nakamura, "Recognition of human driving behaviors based on stochastic symbolization of time series signal," in Proceedings of the IEEE/RSJ International Conference on Intelligent Robots and Systems (IROS '08), pp. 167-172, Nice, France, September 2008.

[26] M. Plöchl and J. Edelmann, "Driver models in automobile dynamics application," Vehicle System Dynamics, vol. 45, no. 7-8, pp. 699-741, 2007.

[27] P. Angkititrakul, T. Ryuta, T. Wakita, K. Takeda, C. Miyajima, and T. Suzuki, "Evaluation of driver-behavior models in realworld car-following task," in Proceedings of the IEEE International Conference on Vehicular Electronics and Safety (ICVES '09), pp. 113-118, Pune, India, November 2009.

[28] K. Igarashi, C. Miyajima, K. Itou, K. Takeda, F. Itakura, and H. Abut, "Biometric identification using driving behavioral signals," in Proceedings of the IEEE International Conference on Multimedia and Expo (ICME '04), vol. 1, pp. 65-68, June 2004.

[29] C. Miyajima, Y. Nishiwaki, K. Ozawa et al., "Driver modeling based on driving behavior and its evaluation in driver identification," Proceedings of the IEEE, vol. 95, no. 2, pp. 427-437, 2007.

[30] Y. Nishiwaki, C. Miyajima, N. Kitaoka, K. Takeda, and K. Itou, "Generation of pedal operation patterns of individual drivers in car-following for personalized cruise control," in Proceedings of the IEEE Intelligent Vehicles Symposium, pp. 823-827, Istanbul, Turkey, June 2007.

[31] P. Angkititrakul, C. Miyajima, and K. Takeda, "Modeling and adaptation of stochastic driver-behavior model with application to car following," in Proceedings of the IEEE Intelligent Vehicles Symposium (IV '11), pp. 814-819, Baden-Baden, Germany, June 2011.

[32] Y. Nishiwaki, C. Miyajima, N. Kitaoka, K. Takeda, and K. Itou, "Generation of pedal operation patterns of individual drivers in car-following for personalized cruise control," in Proceedings of the IEEE Intelligent Vehicles Symposium, pp. 823-827, Istanbul, Turkey, June 2007.

[33] J. Wang, M. Lu, and K. Li, "Characterization of longitudinal driving behavior by measurable parameters," Transportation Research Record, vol. 2185, no. 1, pp. 15-23, 2010. 
[34] J. Wang, R. Chi, L. Zhang, K. Li, and T. Yu, "Study on forward collision warning avoidance algorithm based on driver characteristics adaptation," Journal of Highway and Transportation Research and Development, vol. 26, supplement 1, 2009.

[35] Z. Lei, A vehicle longitudinal driving assistance system based on self-learning method of driver characteristics [Ph.D. thesis], Tsinghua University, 2009.

[36] L. Zhang, S. Li, J. Wang, and K. Li, "Composite driver carfollowing model based on neural network approach," Journal of Tsinghua University: Science and Technology, vol. 11, article 025, 2008.

[37] W. Chang, Research on driving intention identification based on hidden Markov model [Ph.D. thesis], College Automotive Engineering, Jilin University, Changchun, China, 2011.

[38] W. Chang, Z. Changfu, and Z. Hongyu, "Driving intention recognition on cornering based on hidden markov model," SAE-C2010E205, pp. 867-870.

[39] L. He, C. Zong, and C. Wang, "Driving intention recognition and behaviour prediction based on a double-layer hidden Markov model," Journal of Zhejiang University SCIENCE C, vol. 13, no. 3, pp. 208-217, 2012.

[40] C. Zong, X. Yang, and C. Wang, "Driving intentions identification and behaviors prediction in car lane change," Journal of Jilin University: Engineering and Technology Edition, pp. 27-32, 2009.

[41] X. Meng, K. K. Lee, and Y. Xu, "Human driving behavior recognition based on hidden markov models," in IEEE International Conference on Robotics and Biomimetics (ROBIO '06), pp. 274279, Kunming, China, December 2006.

[42] C. S. Papacostas and N. E. Synodinos, "Dimensions of driving behaviour and driver characteristics," Applied Psychology, vol. 37, no. 1, pp. 3-13, 1988.

[43] A. M. Al-Hemoud, R. J. Simmons, and M. M. Al-Asfoor, "Behavior and lifestyle characteristics of male Kuwaiti drivers," Journal of Safety Research, vol. 41, no. 4, pp. 307-313, 2010.

[44] T. Daimon, M. Nishimura, and H. Kawashima, "Study of driver's behavioral characteristics for designing interfaces of in-vehicle navigation systems based on national and regional factors," JSAE Review, vol. 21, no. 3, pp. 379-384, 2000.

[45] A. Wahab, T. G. Wen, and N. Kamaruddin, "Understanding driver behavior using multi-dimensional CMAC Information," in Proceedings of the 6th IEEE International Conference on Communications \& Signal Processing, pp. 1-5, Singapore, December 2007.

[46] T. Wakita, K. Ozawa, C. Miyajima, and K. Takeda, "Parametric versus non-parametric models of driving behavior signals for driver identification," in Audio-and Video-Based Biometric Person Authentication, Lecture Notes in Computer Science, pp. 739-747, Springer, Berlin, Germany, 2005.

[47] O. Raz, H. Fleishman, and I. Mulchadsky, "System and method for vehicle driver behavior analysis and evaluation," U.S. Patent 7389178, 2008.

[48] C. MacAdam, Z. Bareket, P. Fancher, and R. Ervin, "Using neural networks to identify driving style and headway control behavior of drivers," Vehicle System Dynamics, vol. 29, supplement 1, pp. 143-160, 1998.

[49] T. Toledo and T. Lotan, "In-vehicle data recorder for evaluation of driving behavior and safety," Transportation Research Record, vol. 1953, no. 1, pp. 112-119, 2006.

[50] T. Toledo, O. Musicant, and T. Lotan, "In-vehicle data recorders for monitoring and feedback on drivers' behavior," Transportation Research C: Emerging Technologies, vol. 16, no. 3, pp. 320331, 2008.
[51] G. Toledo, Y. Shiftan, and S. Hakkert, "Framework for analysis, and modeling of driving behavior incorporating in-vehicle data recorders," in Proceedings of the 11th World Conference on Transport Research, Berkeley, Calif, USA, 2007.

[52] M. Ueyama, "Driver characteristic using driving monitoring recorder," in Proceedings of the 17th International Technical Conference on the Enhanced Safety of Vehicles Conference, paper 426, 2001.

[53] H. E. Cao, B. Chen, Q. Chen, and J. B. Wang, "Experimental study of the driving-characteristic of occupational drivers and non-occupational drivers," in International Conference on Automobile and Traffic Science, Materials, Metallurgy Engineering (MMAT '12), 2012.

[54] X. Ma and I. Andréasson, "Classification of car following regimes based on clustering analysis," European Transportation Research Arena. In press.

[55] X. Ma and I. Andreasson, "Behavior measurement, analysis, and regime classification in car following," IEEE Transactions on Intelligent Transportation Systems, vol. 8, no. 1, pp. 144-156, 2007.

[56] J. Wu, M. Brackstone, and M. McDonald, "The validation of a microscopic simulation model: a methodological case study," Transportation Research C: Emerging Technologies, vol. 11, no. 6, pp. 463-479, 2003.

[57] S. Theodoridis and K. Koutroumbas, Pattern Recognition, Academic Press, San Diego, Calif, USA, 1999.

[58] G. H. Ball and D. J. Hall, "A clustering technique for summarizing multivariate data," Behavioral Science, vol. 12, no. 2, pp. 153-155, 1967.

[59] M. Ishibashi, M. Okuwa, and M. Akamatsu, "Indices for characterizing driving style and their relevance to car following behavior," in SICE Annual Conference, pp. 1132-1137, Takamatsu, Japan, 2007.

[60] D. Pomerleau, Progress in Neural Network-Based Vision for Autonomous Robot Driving, School of computer science, Carnegie Mellon University, Pittsburg, Pa, USA, 2000.

[61] C. G. Looney, Pattern Recognition Using Neural Networks, Oxford University Press, New York, NY, USA, 1997.

[62] Y. Lin, P. Tang, W. J. Zhang et al., "Artificial neural network modelling of driver handling behaviour in a driver-vehicleenvironment system," International Journal of Vehicle Design, vol. 37, no. 1, pp. 24-45, 2005.

[63] C. G. Quintero M., J. A. O. López, and J. M. P. Rúa, "Intelligent erratic driving diagnosis based on artificial neural networks," in Proceedings of the IEEE ANDESCON Conference (ANDESCON '10), pp. 1-6, Bogotá, Colombia, September 2010.

[64] J. S. Albus, "A new approach to manipulator control: the cerebellar model articulation controller (CMAC)," Journal of Dynamic Systems, Measurement and Control, vol. 97, no. 3, pp. 220-227, 1975.

[65] X. Zou and D. Levinson, "Modeling intersection driving behaviours: a hidden markov model approach-I," Journal of the Transportation Research Board, pp. 19-23, 2006.

[66] L. An, H. Zhencheng, and C. Hui, "Recognition and analysis on highway overtaking behavior based on Gaussian mixturehidden markov model," Automotive Engineering, vol. 32, no. 7, pp. 630-634, 2010.

[67] A. Sathyanarayana, P. Boyraz, and J. H. L. Hansen, "Driver behavior analysis and route recognition by hidden Markov models," in Proceedings of the IEEE International Conference on Vehicular Electronics and Safety (ICVES '08), pp. 276-281, Columbus, Ohio, USA, September 2008. 
[68] Y. Wang, J. Song, and X. Li., "Study on inference of driver's intentions based on fuzzy reasoning," Journal of Highway and Transportation Research and Development, vol. 12, article 029, 2005.

[69] T. Hessburg and M. Tomizuka, "Fuzzy logic control for lateral vehicle guidance," IEEE Control Systems, vol. 14, no. 4, pp. 55-63, 1994.

[70] A. Sathyanarayana, P. Boyraz, and J. H. L. Hansen, "Information fusion for robust "context and driver aware" active vehicle safety systems," Information Fusion, vol. 12, no. 4, pp. 293-303, 2011.

[71] U. Kiencke, R. Majjad, and S. Kramer, "Modeling and performance analysis of a hybrid driver model," Control Engineering Practice, vol. 7, no. 8, pp. 985-991, 1999.

[72] G. N. Bifulco, F. Galante, L. Pariota, and M. Russo-Spena, "Identification of driving behaviors with computer-aided tools," in Proceedings of the 6th UKSim/AMSS European Symposium on Computer Modeling and Simulation (EMS '12), vol. 2012, pp. 331336, Valetta, Malta, November 2012.

[73] G. N. Bifulco, F. Simonelli, and R. di Pace, "Experiments toward an human-like adaptive cruise control," in Proceedings of the IEEE Intelligent Vehicles Symposium, pp. 919-924, Eindhoven, The Netherlands, June 2008.

[74] G. Zhao, C. Peng, and X. Wang, "Intelligent control for AMT based on driver's intention and ANFIS decision-making," in Proceedings of the 7th World Congress on Intelligent Control and Automation (WCICA '08), pp. 1-6, Chongqing, China, June 2008.

[75] E. U. Warriach, M. Aiello, and K. A. Tei, "Machine learning approach for identifying and classifying faults in wireless sensor Network," in Proceedings of the IEEE 15th International Conference on Computational Science and Engineering (CSE '12), pp. 618-625, Nicosia, Cyprus, December 2012.

[76] C. M. Bishop, Neural Networks for Pattern Recognition, Oxford university press, New York, NY, USA, 1995.

[77] http://www.eepw.com.cn/article/2506.htm.

[78] Z. Xia and L. Chen, "A moving object detection algorithm based on Gaussian mixture model," Journal of Hangzhou Dianzi University, vol. 2, article 016, 2011.

[79] T. Imamura, H. Yamashita, M. D. R. bin Othman, Z. Zhang, and T. Miyake, "Driving behavior classification and driver sensing based on vehicle steering wheel operations," in Proceedings of the SICE Annual Conference, pp. 2714-2718, Tokyo, Japan, August 2008.

[80] T. Shiiba and Y. Suda, "Evaluation of driver's behavior with multibody-based driving simulator," Multibody System Dynamics, vol. 17, no. 2-3, pp. 195-208, 2007.

[81] C. Miyajima, Y. Nishiwaki, K. Ozawa, T. Wakita, K. Itou, and K. Takeda, "Cepstral analysis of driving behavioral signals for driver identification," in Proceedings of the IEEE International Conference on Acoustics, Speech and Signal Processing (ICASSP '06), pp. V921-V924, Toulouse, France, May 2006.

[82] X. Ma and I. Andréasson, "Driver reaction time estimation from real car following data and application in GM-type model evaluation," in Proceedings of the 85th TRB Annual Meeting, pp. 1-19, 2006.

[83] B. Cheng, T. Taniguchi, T. Hatano et al., "Study on the characteristics of driver behaviors in car following," Transactions of Society of Automotive Engineers of Japan, vol. 38, no. 1, pp. 171176, 2004.

[84] T. Wakita, K. Ozawa, C. Miyajima et al., "Driver identification using driving behavior signals," IEICE Transactions on Information and Systems D, vol. E89, no. 3, pp. 1188-1194, 2006.
[85] A. Wahab, T. C. Keong, H. Abut, and K. Takeda, "Driver recognition system using FNN and statistical methods," in Advances for In-Vehicle and Mobile Systems, pp. 11-23, Springer, New York, NY, USA, 2007.

[86] L. Zhang, J. Wang, K. Li et al., "A study on collision warning/avoidance algorithms based on driver's behaviors during car-following," Automotive Engineering, vol. 28, no. 4, pp. 351$375,2006$. 


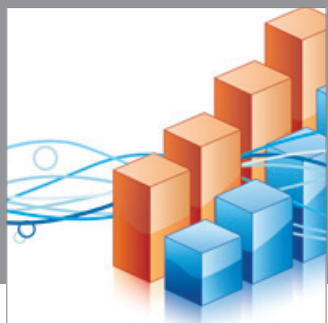

Advances in

Operations Research

mansans

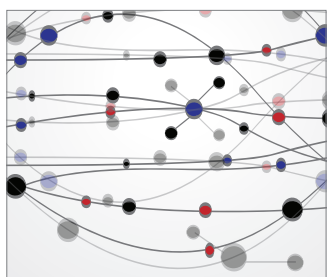

The Scientific World Journal
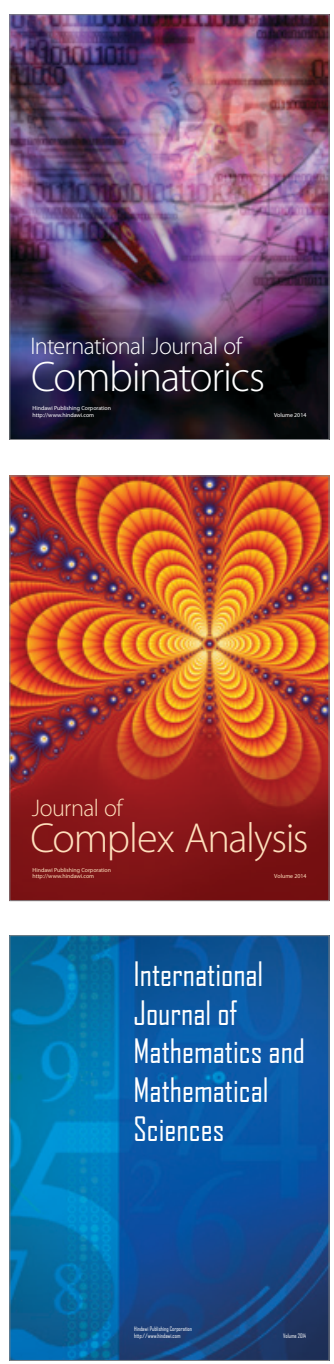
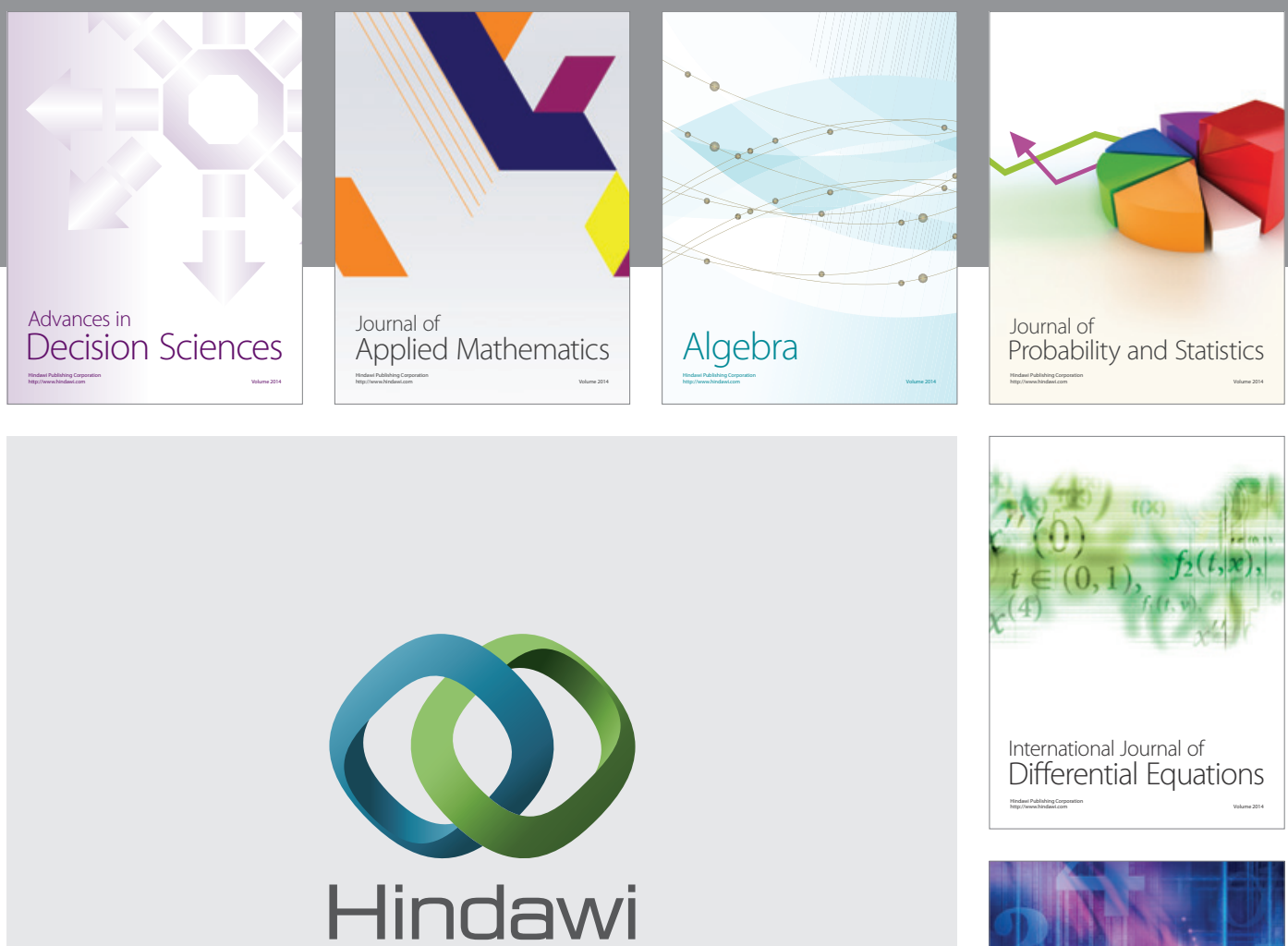

Submit your manuscripts at http://www.hindawi.com
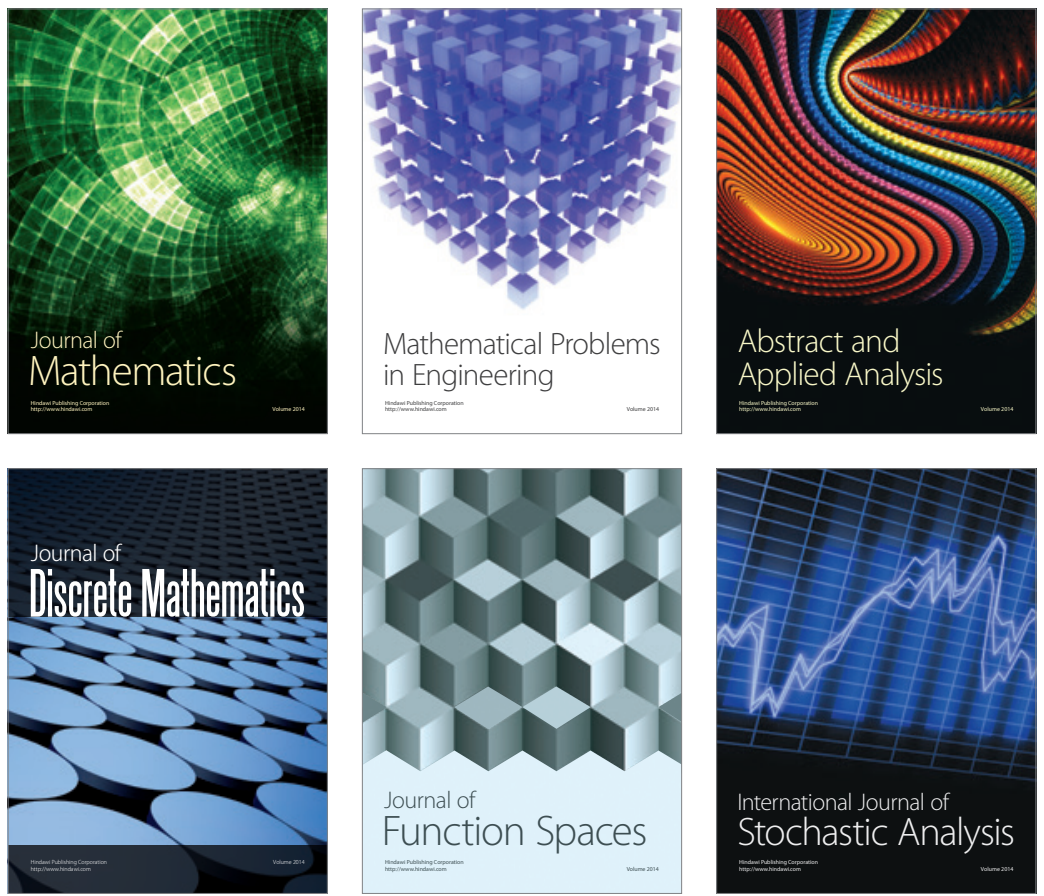

Journal of

Function Spaces

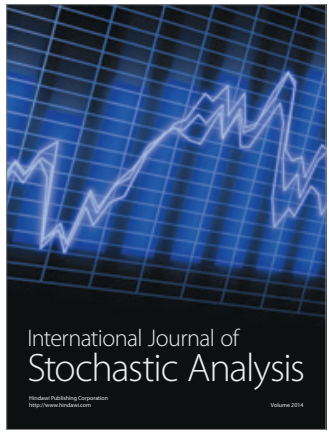

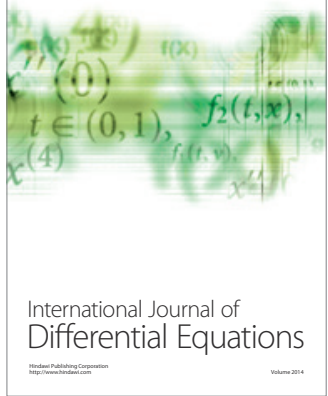
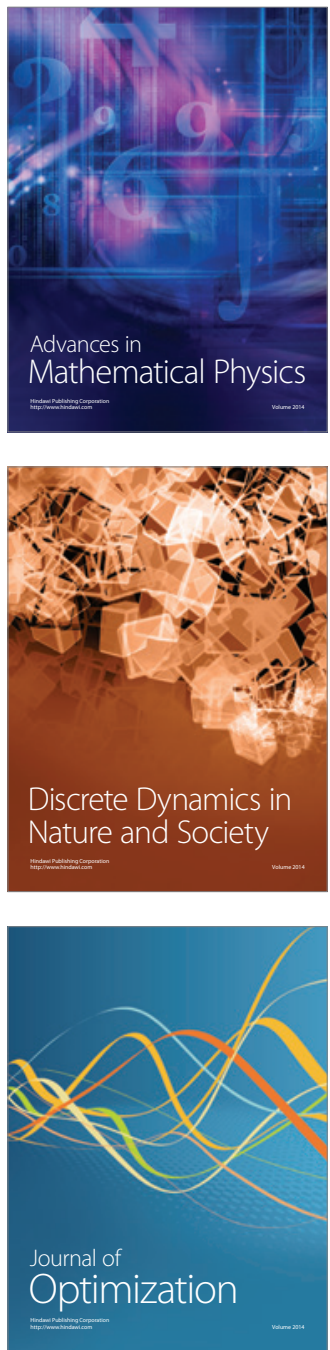